\section{O impacto odontológico no desempenho diário dos trabalhadores do Departamento Municipal de Limpeza Urbana de Porto Alegre, Rio Grande do Sul, Brasil}

\author{
The impact of oral health on daily performance of \\ municipal waste disposal workers in Porto Alegre, \\ Rio Grande do Sul State, Brazil
}

\author{
${ }^{1}$ Faculdade de Odontologia, \\ Universidade Federal do \\ Rio Grande do Sul, \\ Porto Alegre, Brasil. \\ Correspondência \\ A. S. Gomes \\ Faculdade de Odontologia, \\ Universidade Federal do \\ Rio Grande do Sul. \\ Av. Ipiranga 3491, apto. 711 \\ Porto Alegre, RS \\ 90610-001, Brasil. \\ andreag@terra.com.br
}

\begin{abstract}
This study aimed to investigate the prevalence of oral health impact on daily performance in Brazilian adults. 276 civil servants 35 to 44 years of age from the Public Works and Waste Disposal Department of Porto Alegre, in southern Brazil, were interviewed and clinically examined. Oral Impacts on Daily Performance (OIDP) was used to evaluate the impact of oral health status on daily performance. $73.6 \%$ of all subjects had at least one daily performance affected by an oral impact in the previous six months. The most commonly affected performance was eating (48.6\%), while the most common symptoms were discomfort (40.6\%) and dissatisfaction with one's appearance (31.5\%). Missing teeth (21.7\%) and toothache (20.7\%) were recognized as the main causes of oral impacts on daily performance. OIDP was useful for measuring (physically, psychologically, and socially) the oral impacts on daily performance.
\end{abstract}

Oral Health; Quality of Life; Indicators; Health Surveys
Andréa Silveira Gomes 1

Claides Abegg 1

\section{Introdução}

Os problemas de saúde bucal têm sido cada vez mais reconhecidos como importantes causadores de impacto negativo no desempenho diário e na qualidade de vida dos indivíduos e da sociedade. O relatório da Organização Mundial da Saúde (OMS) ${ }^{1}$ reconheceu que as doenças bucais causam dor, sofrimento, constrangimentos psicológicos e privações sociais, acarretando prejuízos em nível individual e coletivo. A saúde bucal, no contexto das definições contemporâneas de saúde, é entendida como uma dentição confortável, funcional, com uma aparência que permite aos indivíduos desempenharem a sua função social e as suas atividades diárias sem transtornos físicos, psicológicos ou sociais 2,3 .

Apesar do reconhecimento da importância dos aspectos sociais e psicológicos na determinação da doença, amplamente debatidos 4,5, a odontologia continua empregando, quase que exclusivamente, índices biológicos na avaliação e determinação das necessidades de tratamento e apreciação de programas de saúde bucal. O índice de dentes cariados, perdidos e obturados (CPOD) e o índice periodontal comunitário (IPC) são recomendados pela OMS ${ }^{6}$ para a avaliação das duas patologias bucais mais prevalentes: a cárie e a doença periodontal. Uma das limitações desses índices é o fato de não considerarem a percepção subjetiva do indivíduo em relação à saúde bucal e de não avaliarem a maneira co- 
mo a saúde bucal afeta a vida diária 7. Contudo, instrumentos de aferição capazes de englobar os aspectos psicológicos e sociais, por intermédio da autopercepção e do levantamento dos impactos causados na qualidade de vida, têm sido desenvolvidos e validados por diversos autores $7,8,9,10,11$.

Os indicadores sócio-dentais conseguem mensurar o grau em que as doenças bucais interferem no funcionamento normal e desejável do indivíduo, desde os aspectos funcionais (como, por exemplo, comer), os psicológicos (como humor, irritação) até os sociais (como freqüentar a escola, trabalhar, desempenhar obrigações familiares). O uso de indicadores sócio-dentais, baseados na autopercepção e nos impactos odontológicos, oferece vantagens importantes para o planejamento e provisão dos serviços odontológicos, e a principal é a mudança na ênfase de aspectos puramente biológicos para aspectos psicológicos e sociais 10 .

O Oral Impacts on Daily Performances OIDP 12 é um indicador sócio-dental baseado conceitualmente no International Classification of Impairments, Disabilities and Handicaps 13, modificado por Locker para o seu uso em odontologia 14. Mediante a avaliação da freqüência e da severidade dos impactos que afetam o desempenho diário dos indivíduos, o OIDP fornece um escore de impacto individual 8 . A classificação da severidade dá um peso à importância relativa do impacto odontológico percebido pelo indivíduo ${ }^{10}$. Além dos aspectos já mencionados, também são questionados os problemas bucais e os sintomas percebidos pelos sujeitos como causadores de impacto, a fim de relacioná-lo à condição clínica, o que torna o OIDP mais consistente para ser utilizado na avaliação das necessidades de tratamento 8 .

Considerando-se a pequena literatura disponível sobre a utilização de indicadores sócio-dentais no Brasil, bem como a relevância que esse enfoque nos aspectos psicológicos e sociais trará para uma nova visão da relação entre saúde bucal e qualidade de vida, faz-se importante a realização de estudos que incorporem e demonstrem essa nova forma de avaliação das condições de saúde bucal e, ainda, proporcionem o conhecimento da prevalência do impacto da saúde bucal no desempenho diário da população brasileira.

O principal objetivo deste estudo foi investigar a prevalência do impacto bucal no desempenho diário em adultos brasileiros. Além disso, avaliou-se a associação do impacto odontológico no desempenho diário com as variáveis sóciodemográficas e clínicas.

\section{Material e método}

Este estudo transversal foi realizado com uma amostra representativa dos 728 funcionários do Departamento Municipal de Limpeza Urbana de Porto Alegre, da faixa etária de 35 a 44 anos, em 2005.

Através do cálculo amostral (nível de confiança $=95 \%$; prevalência esperada $=50 \%$; erro amostral previsto $=5 \%$ ), foi definida uma amostra mínima de 251 pessoas. O tamanho da amostra foi aumentado em $10 \%$ para compensar possíveis perdas, portanto, no total, 276 funcionários participaram da entrevista e do exame clínico. Ao final do estudo, houve perda de cinco indivíduos, que não foram repostos.

Previamente ao sorteio, a população-alvo foi organizada conforme a ocupação, dividindo-se em funcionários da área administrativa e funcionários da área operacional, a fim de garantir diferentes níveis de escolaridade e de renda na amostra do estudo.

Aplicou-se um questionário composto pelas variáveis sócio-demográficas e pelo índice OIDP 12, índice que já foi validado e utilizado numa população brasileira 15,16 .

Os indivíduos foram questionados se "nos últimos seis meses algum problema com a sua saúde bucal causou dificuldades ou prejuizos para comer e apreciar a comida, falar e pronunciar com clareza, higienizar os dentes, dormir e relaxar, sorrir, dar risadas e mostrar os dentes sem ficar envergonhado, manter um estado emocional equilibrado sem ficar irritado, desempenhar o trabalho principal ou o papel social, elou gostar de ter contato com as pessoas". As respostas possíveis foram sim e não.

O OIDP originalmente contava com nove desempenhos para serem avaliados. Todavia, constatou-se que a avaliação do desempenho desenvolver uma atividade física estava redundante com a questão desempenhar o trabalho principal ou o papel social. Sendo assim, desempenhar uma atividade física ou trabalho doméstico foi removido da versão final do índice, passando este a ser denominado por alguns autores de OIDP abreviado 14 e OIDP modificado 17 . Neste estudo foi utilizada a nova versão, composta por oito desempenhos.

Os desempenhos afetados foram classificados de acordo com a freqüência de impacto através do padrão de ocorrência: 1 - menos de uma vez ao mês, ou intervalo de até cinco dias no total; 2 - uma ou duas vezes ao mês, ou intervalo de até 15 dias no total; 3 - uma ou duas vezes por semana, ou intervalo de até trinta dias no total; 4 - três ou quatro vezes por semana, ou intervalo de até três meses no total; 5 - todo ou quase todo dia, 
ou intervalo de mais de três meses no total. Os desempenhos afetados também foram classificados de acordo com o grau de severidade atribuído pelo indivíduo: 0 - nenhuma gravidade; 1 - muito pouco grave; 2 - pouco grave; 3 - gravidade moderada; 4 - muito grave; 5 - extremamente grave. Para cada impacto relatado, foram registrados o principal sintoma (dor, desconforto, limitação na função, insatisfação com a aparência ou outro) e o problema bucal responsável pelo impacto.

O exame clínico foi realizado por uma cirurgiã-dentista treinada e calibrada segundo os critérios da OMS 6, no mesmo dia da entrevista, utilizando o índice CPOD e iluminação artificial (lanterna de mão).

O estudo-piloto foi realizado com vinte funcionários da mesma faixa etária que não foram sorteados para o estudo principal. Após o término da coleta de dados, foram realizados vinte reexames $(7,2 \%$ da amostra) e, a cada dez indivíduos examinados, o último foi reexaminado. No exercício de calibração, os valores do kappa intra-examinador foram de 0,91 para cárie de coroa e de 0,83 para cárie de raiz. Nos reexames, os valores do kappa intra-examinador foram de 0,92 para cárie de coroa e de 0,85 para cárie de raiz.

Os dados foram analisados utilizando-se o SPSS para Windows, versão 8.0 (SPSS Inc., Chicago, Estados Unidos).

A análise estatística compreendeu a análise descritiva das características gerais da população estudada, mediante a apresentação das freqüências absolutas e relativas. A seguir, foram realizadas análises bivariadas entre as variáveis sócio-demográficas e clínicas e o impacto odontológico nos desempenhos físicos, psicológicos e sociais diários. O nível de significância adotado foi $\alpha=0,05$. Foram utilizados os testes qui-quadrado de Pearson e Exato de Fisher. As variáveis sócio-demográficas utilizadas foram sexo (feminino/masculino), escolaridade (medida em anos de estudo e estratificada em uma categoria de até seis anos de estudo e outra de sete anos ou mais de estudo) e nível de renda pessoal (medida em categorias de salários mínimos e estratificada em uma categoria de até quatro salários mínimos e outra, com valores superiores a quatro salários mínimos). A variável clínica CPOD foi dividida em tercis, de acordo com a distribuição da freqüência: CPOD baixo, CPOD médio e CPOD alto. A divisão desse índice foi realizada de acordo com o percentual de pessoas, de modo a formar grupos de tamanhos homogêneos para serem comparados.

A aprovação do Comitê de Ética em Pesquisa da Universidade Federal do Rio Grande do Sul, bem como da direção do departamento municipal de limpeza urbana de Porto Alegre, antece- deram a fase de coleta de dados. Os funcionários receberam, antes do início da entrevista, uma explicação sobre o objetivo da pesquisa e sobre o termo de consentimento informado, que foi lido pelo anotador e assinado pelos participantes.

\section{Resultados}

Dos 276 indivíduos da amostra, 201 (72,8\%) eram do sexo masculino. A maioria dos entrevistados $(54,3 \%)$ declarou ter ensino fundamental incompleto, e a média de anos estudados foi de 7,34 $(\mathrm{DP}=4,77)$. Em relação à renda pessoal, do total da amostra, 4,7\% declararam receber até dois salários mínimos; $51,1 \%$, até quatro salários mínimos; $39,9 \%$, entre quatro e dez salários mínimos, e os demais recebem valor superior a dez salários mínimos.

Dos 276 indivíduos examinados, 203 (73,6\%) relataram pelo menos um desempenho, ou atividade diária, afetado por problemas odontológicos nos últimos seis meses. O desempenho diário mais afetado foi o físico comer e apreciar a comida (48,6\%), seguido pelo também físico higienizar os dentes $(38,4 \%)$ e pelo psicológico sorrir e mostrar os dentes sem ficar envergonhado $(37,3 \%)$. Verificou-se que a maioria dos impactos odontológicos apresentava alta freqüência (mo$\mathrm{da}=5)$ e alta severidade $($ moda $=5)($ Tabela 1$)$.

O desconforto (40,6\%), a insatisfação com a aparência (31,5\%) e a dor (31,2\%) foram os sintomas prevalentes entre as pessoas que relataram pelo menos um desempenho diário afetado por problemas bucais (Tabela 2).

Os principais sintomas e problemas relacionados ao impacto odontológico no desempenho diário podem ser verificados na Tabela 3 .

Houve associação significativa entre os desempenhos diários afetados e as variáveis sóciodemográficas, sendo sexo a variável que mais apresentou associação com os desempenhos físicos, psicológicos e sociais (Tabela 4).

Em relação às condições de saúde bucal, a amostra examinada apresentou CPOD médio de 16,45 (DP = 6,70), distribuídos em cariados ( édia $=1,61 ; \mathrm{DP}=2,43$ ), perdidos (média $=7,85$; $\mathrm{DP}=6,20$ ) e obturados (média $=6,99 ; \mathrm{DP}=6,10$ ). Os participantes apresentaram em média 24 dentes presentes; apenas dois indivíduos eram desdentados totais.

Quando os escores totais de CPOD foram categorizados em baixo, médio e alto e analisados em relação aos desempenhos que sofreram impactos odontológicos, observou-se associação significativa entre o CPOD com desempenhos nas dimensões físicas, psicológicas e sociais (Tabela 5). 
Prevalência, freqüência e severidade dos impactos bucais nos desempenhos diários.

\begin{tabular}{|c|c|c|c|c|c|c|}
\hline \multirow[t]{2}{*}{ Desempenhos diários } & \multicolumn{2}{|c|}{$\begin{array}{l}\text { Pessoas afetadas } \\
\qquad(N=276)\end{array}$} & \multicolumn{2}{|c|}{$\begin{array}{l}\text { Freqüência } \\
(1-5)\end{array}$} & \multicolumn{2}{|c|}{$\begin{array}{c}\text { Severidade } \\
(0-5)\end{array}$} \\
\hline & $\mathbf{n}$ & $\%$ & Mediana & Moda & Mediana & Moda \\
\hline \multicolumn{7}{|l|}{ Desempenho físico } \\
\hline 1. Comer e apreciar a comida & 134 & 48,6 & 3 & 5 & 3 & 5 \\
\hline 2. Falar e pronunciar com clareza & 51 & 18,5 & 3 & 5 & 4 & 5 \\
\hline 3. Higienizar os dentes & 106 & 38,4 & 5 & 5 & 3 & 3 \\
\hline \multicolumn{7}{|l|}{ Desempenho psicológico } \\
\hline 4. Dormir e relaxar & 49 & 17,8 & 4 & 5 & 4 & 5 \\
\hline 5. Sorrir, dar risadas e mostrar os dentes sem ficar envergonhado & 103 & 37,3 & 5 & 5 & 4 & 5 \\
\hline 6. Manter um estado emocional equilibrado sem ficar irritado & 63 & 22,8 & 4 & 5 & 4 & 5 \\
\hline \multicolumn{7}{|l|}{ Desempenho social } \\
\hline 7. Desempenhar o trabalho principal ou o papel social & 46 & 16,7 & 3 & 5 & 4 & 5 \\
\hline 8. Gostar de ter contato com as pessoas & 69 & 25,0 & 5 & 5 & 4 & 5 \\
\hline Total & 203 & 73,6 & & & & \\
\hline
\end{tabular}

Tabela 2

Principais sintomas e problemas bucais que afetam os desempenhos diários $(N=276)$

\begin{tabular}{lll}
\hline & $\mathbf{n}$ & $\%$ \\
\hline Principais sintomas & & \\
Desconforto & 112 & 40,6 \\
Insatisfação com a aparência & 87 & 31,5 \\
Dor & 86 & 31,2 \\
Limitação na função & 82 & 29,7 \\
Principais problemas bucais & & \\
Falta de dente & 60 & 21,7 \\
Dor de dente & 57 & 20,7 \\
Dentadura solta & 29 & 10,5 \\
Obturação quebrada ou cor diferente do dente & 26 & 9,4 \\
Hemorragias gengivais & 24 & 8,7 \\
Gosto desagradável & 19 & 6,9 \\
Afastamento gengival & 17 & 6,2 \\
Úlcera bucal & 17 & 6,2
\end{tabular}

\section{Discussão}

A alta prevalência $(73,6 \%)$ de impactos bucais no desempenho diário na população estudada é consistente com a prevalência encontrada por outros autores que utilizaram o índice OIDP: 73,6\% na faixa etária de 35 a 44 anos 12; $51 \%$ em indivíduos com uma média de idade de 26,4 anos $14 ; 69,1 \%$ em indivíduos de 19 a 74 anos 18 e $52,8 \%$ em idosos entre 60 e 74 anos 19 .
Salienta-se que, de acordo com a literatura, a faixa etária de 35 a 44 anos é a que apresenta maior prevalência de impacto. Isso pode estar relacionado a uma maior presença de necessidades de tratamento não atendidas, em virtude da dificuldade de acesso ao tratamento clínico deste grupo etário.

Dados do Projeto SB Brasil 2003 20, que avaliou as condições de saúde bucal da população brasileira nos anos de 2002 e 2003, demonstraram que, para as faixas etárias de 15-19 e de 35-44, o CPOD foi de 6,17 e de 20,13, respectivamente. O componente cariado foi responsável por 2,79 e 2,68 do total do índice, enquanto o componente perdido correspondeu ao percentual de 0,89 e 13,23 do total, respectivamente. Esses dados retratam o número expressivo de dentes atingidos pela doença cárie e indicam o crescimento da necessidade de tratamento na faixa etária de 3544 anos, o que pode ser reflexo de maior prevalência de impacto odontológico para esse estrato etário.

O desempenho diário mais afetado neste estudo foi o desempenho físico comer e apreciar a comida, resultado também verificado por outros autores 14,17,19. Em relação à freqüência diária de impacto, observou-se que a mais comum foi todos ou quase todos os dias para todos os desempenhos. Em relação à severidade, verificou-se que, com exceção do desempenho físico higienizar os dentes, os impactos foram classificados como extremamente graves. Outros estudos encontraram freqüência e severidade dos impactos mais baixos 12,14,19. Constatou-se que a 
Principais sintomas e problemas bucais que afetaram os desempenhos diários.

\begin{tabular}{|c|c|c|c|c|c|c|}
\hline \multirow[t]{2}{*}{ Desempenhos diários } & \multicolumn{3}{|c|}{ Principais sintomas } & \multicolumn{3}{|c|}{ Principais problemas bucais } \\
\hline & & $\mathrm{n}$ & $\%$ & & $\mathrm{n}$ & $\%$ \\
\hline \multicolumn{7}{|l|}{ Desempenho físico } \\
\hline \multirow[t]{3}{*}{ 1. Comer e apreciar a comida $(N=134)$} & Limitação na função & 51 & 38,1 & Dor de dente & 38 & 28,4 \\
\hline & Dor & 43 & 32,1 & Dentadura solta & 22 & 16,4 \\
\hline & & & & Falta de dente & 18 & 13,4 \\
\hline \multirow[t]{3}{*}{ 2. Falar e pronunciar com clareza $(\mathrm{N}=51)$} & Limitação na função & 22 & 43,1 & Falta de dente & 16 & 31,4 \\
\hline & Desconforto & 15 & 29,4 & Dentadura solta & 14 & 27,5 \\
\hline & & & & Úlcera bucal & 7 & 13,7 \\
\hline \multirow[t]{3}{*}{ 3. Higienizar os dentes $(\mathrm{N}=106)$} & Desconforto & 48 & 45,3 & Dor de dente & 22 & 20,8 \\
\hline & Dor & 35 & 33,0 & Hemorragias gengivais & 22 & 20,8 \\
\hline & & & & Afastamento gengival & 13 & 12,3 \\
\hline \multicolumn{7}{|l|}{ Desempenho psicológico } \\
\hline \multirow[t]{2}{*}{ 4. Dormir e relaxar $(\mathrm{N}=49)$} & Dor & 26 & 53,1 & Dor de dente & 17 & 34,7 \\
\hline & Desconforto & 15 & 30,6 & & & \\
\hline 5. Sorrir, dar risadas e mostrar os dentes & Insatisfação com a aparência & 69 & 67,0 & Falta de dente & 44 & 42,7 \\
\hline \multirow[t]{2}{*}{ sem ficar envergonhado $(N=103)$} & Desconforto & 25 & 24,3 & Dentadura solta & 15 & 14,6 \\
\hline & & & & Cor dos dentes & 14 & 13,6 \\
\hline 6. Manter um estado emocional equilibrado & Desconforto & 18 & 28,6 & Dor de dente & 14 & 22,2 \\
\hline \multirow[t]{2}{*}{ sem ficar irritado $(\mathrm{N}=63)$} & Dor & 17 & 27,0 & Falta de dente & 11 & 17,5 \\
\hline & Insatisfação com a aparência & 17 & 27,0 & Dentadura solta & 8 & 12,7 \\
\hline \multicolumn{7}{|l|}{ Desempenho social } \\
\hline 7. Desempenhar o trabalho principal & Dor & 19 & 41,3 & Dor de dente & 15 & 32,6 \\
\hline \multirow[t]{2}{*}{ ou o papel social $(\mathrm{N}=46)$} & Insatisfação com a aparência & 12 & 26,1 & Falta de dente & 10 & 21,7 \\
\hline & & & & Dentadura solta & 5 & 10,9 \\
\hline 8. Gostar de ter contato com & Insatisfação com a aparência & 36 & 52,2 & Falta de dente & 24 & 34,8 \\
\hline as pessoas $(\mathrm{N}=69)$ & Desconforto & 16 & 23,2 & Gosto desagradável & 16 & 23,2 \\
\hline
\end{tabular}

alta freqüência e a alta severidade dos impactos estão relacionadas com o desconforto, a insatisfação com a aparência e a dor, causados pelos problemas dor de dente e falta de dentes.

Alguns autores também encontraram dor e desconforto como os principais sintomas dos impactos bucais e dor de dente, como o principal problema causador de impacto bucal 12 , ao passo que outros verificaram a falta de dentes como o principal problema de saúde bucal 19. De acordo com esses estudos, a dor de dente está mais relacionada com a presença de dentes, enquanto a limitação na função e a insatisfação com a aparência estão mais relacionadas com a falta de dentes. A dor de dente pode incluir, na avaliação da pessoa leiga, comprometimentos bucais mais amplos do que aqueles originados pela cárie dentária 12 . Estudos mostram que dor de dente $\mathrm{e}$ limitação na função estão mais fortemente associados ao impacto no desempenho diário do que a presença clínica de doença 21 .

No presente estudo, verificou-se associação significativa entre sexo e a maioria dos impactos nos desempenhos diários, o mesmo ocorrendo em outros estudos, em que as mulheres relataram maior impacto odontológico do que os homens ${ }^{22}$. A diferença significativa entre homens e mulheres pode ser atribuída às diferenças de percepção sobre saúde e o valor da saúde bucal entre os dois sexos, além de condições hormonais e de maior prevalência de doenças sistêmicas que influenciam na saúde bucal das mulheres 22 .

Também houve associação significativa entre escolaridade e renda e o impacto em alguns 
Tabela 4

Relação de associação entre desempenho diário e variáveis sócio-demográficas. Freqüências absolutas e relativas dos indivíduos que relataram impacto odontológico ( $\mathrm{N}=276)$.

\begin{tabular}{|c|c|c|c|c|c|c|c|c|c|}
\hline \multirow[t]{2}{*}{ Desempenhos diários } & \multicolumn{3}{|c|}{ Sexo } & \multicolumn{3}{|c|}{ Escolaridade (anos) } & \multicolumn{3}{|c|}{ Renda (salários mínimos) } \\
\hline & $\begin{array}{l}\text { Feminino } \\
\text { n (\%) }\end{array}$ & $\begin{array}{c}\text { Masculino } \\
\text { n (\%) }\end{array}$ & valor $\mathrm{p}$ & $\begin{array}{c}\leq 6 \\
\mathrm{n}(\%)\end{array}$ & $\begin{array}{l}>6 \\
\mathrm{n}(\%)\end{array}$ & valor $\mathrm{p}$ & $\begin{array}{c}\leq 4 \\
n(\%)\end{array}$ & $\begin{array}{c}>4 \\
\mathrm{n}(\%)\end{array}$ & valor $\mathrm{p}$ \\
\hline \multicolumn{10}{|l|}{ Desempenho físico } \\
\hline 1. Comer e apreciar a comida & $43(57,3)$ & $91(45,2)$ & 0,050 & $72(51,4)$ & $62(45,6)$ & 0,198 & $65(46,1)$ & $69(51,1)$ & 0,238 \\
\hline 2. Falar e pronunciar com clareza & $17(22,6)$ & $34(16,9)$ & 0,178 & $33(23,6)$ & $18(13,2)$ & 0,019 & $22(15,6)$ & $29(21,5)$ & 0,135 \\
\hline 3. Higienizar os dentes & $37(49,3)$ & $69(34,3)$ & 0,017 & $54(38,6)$ & $52(38,2)$ & 0,527 & $53(37,6)$ & $53(39,3)$ & 0,436 \\
\hline \multicolumn{10}{|l|}{ Desempenho psicológico } \\
\hline 4. Dormir e relaxar & $20(26,6)$ & $29(14,4)$ & 0,016 & $29(20,7)$ & $20(14,7)$ & 0,125 & $31(22,0)$ & $18(13,3)$ & 0,042 \\
\hline $\begin{array}{l}\text { 5. Sorrir, dar risadas e mostrar os } \\
\text { dentes sem ficar envergonhado }\end{array}$ & $35(46,6)$ & $68(33,8)$ & 0,035 & $56(40,0)$ & $47(34,6)$ & 0,209 & $61(43,3)$ & $42(31,1)$ & 0,025 \\
\hline $\begin{array}{l}\text { 6. Manter um estado emocional } \\
\text { equilibrado sem ficar irritado }\end{array}$ & $25(33,3)$ & $38(18,9)$ & 0,010 & $38(27,1)$ & $25(18,4)$ & 0,056 & $32(22,7)$ & $31(23,0)$ & 0,536 \\
\hline \multicolumn{10}{|l|}{ Desempenho social } \\
\hline $\begin{array}{l}\text { 7. Desempenhar o trabalho } \\
\text { principal ou o papel social }\end{array}$ & $19(25,3)$ & $27(13,4)$ & 0,017 & $25(17,9)$ & $21(15,4)$ & 0,353 & $28(19,9)$ & $18(13,3)$ & 0,098 \\
\hline $\begin{array}{l}\text { 8. Gostar de ter contato com } \\
\text { as pessoas }\end{array}$ & $23(30,6)$ & $46(22,8)$ & 0,121 & $40(28,6)$ & $29(21,3)$ & 0,105 & $41(29,1)$ & $28(20,7)$ & 0,072 \\
\hline
\end{tabular}

Tabela 5

Relação entre desempenhos diários e variáveis clínicas em associação ao teste qui-quadrado. Freqüências absolutas e relativas dos indivíduos que relataram impacto odontológico $(\mathrm{N}=276)$.

\begin{tabular}{|c|c|c|c|c|c|c|c|}
\hline \multirow[t]{3}{*}{ Desempenhos diários } & & \multicolumn{6}{|c|}{ CPOD * } \\
\hline & \multicolumn{2}{|c|}{ Baixo } & \multicolumn{2}{|c|}{ Médio } & \multicolumn{2}{|c|}{ Alto } & \multirow[t]{2}{*}{ valor $\mathrm{p}$} \\
\hline & $\mathrm{n}$ & $\%$ & $\mathrm{n}$ & $\%$ & $\mathbf{n}$ & $\%$ & \\
\hline \multicolumn{8}{|l|}{ Desempenho físico } \\
\hline 1. Comer e apreciar a comida & 33 & 39,8 & 44 & 44,9 & 57 & 60,0 & 0,018 \\
\hline 2. Falar e pronunciar com clareza & 10 & 12,0 & 14 & 14,3 & 27 & 28,4 & 0,008 \\
\hline 3. Higienizar os dentes & 32 & 38,6 & 36 & 36,7 & 38 & 40,0 & 0,896 \\
\hline \multicolumn{8}{|l|}{ Desempenho psicológico } \\
\hline 4. Dormir e relaxar & 10 & 12,0 & 17 & 17,3 & 22 & 23,2 & 0,152 \\
\hline $\begin{array}{l}\text { 5. Sorrir, dar risadas e mostrar os } \\
\text { dentes sem ficar envergonhado }\end{array}$ & 18 & 21,7 & 34 & 34,7 & 51 & 53,7 & 0,001 \\
\hline $\begin{array}{l}\text { 6. Manter um estado emocional } \\
\text { equilibrado sem ficar irritado }\end{array}$ & 12 & 14,5 & 16 & 18,4 & 33 & 34,7 & 0,002 \\
\hline \multicolumn{8}{|l|}{ Desempenho social } \\
\hline $\begin{array}{l}\text { 7. Desempenhar o trabalho principal } \\
\text { ou o papel social }\end{array}$ & 7 & 8,4 & 15 & 15,3 & 24 & 25,3 & 0,010 \\
\hline 8. Gostar de ter contato com as pessoas & 15 & 18,1 & 24 & 24,5 & 30 & 31,6 & 0,115 \\
\hline
\end{tabular}

* O escore total do CPOD foi dividido em três categorias, baixo (0-12), médio (13-19) e alto (20-32), de acordo com a distribuição das freqüências. 
desempenhos diários. Alguns estudos têm demonstrado que a escolaridade e a renda vêm influenciando a autopercepção de saúde bucal e o relato de impacto odontológico na qualidade de vida; têm demonstrado, ainda, que baixo nível de escolaridade e baixa renda têm relação com uma maior prevalência de impactos bucais $17,19,23,24,25$.

Verificou-se ainda, neste estudo, relação entre o impacto percebido e a condição clínica dos dentes, achado confirmado por outros trabalhos 9,23,26. A associação significativa entre as categorias de CPOD baixo, médio e alto da presente pesquisa também foi verificada em adultos brasileiros da mesma faixa etária, utilizando-se, porém, outro indicador sócio-dental 27. Esse achado é importante, pois a maioria dos estudos utiliza o índice CPOD e, com base em uma categorização em grupos de CPOD baixo, médio e alto, a partir das freqüências, poderão ser identificados os grupos mais vulneráveis a impactos nos desempenhos físicos, psicológicos e sociais.

\section{Conclusões}

Os resultados deste trabalho permitiram concluir que na população estudada: (1) a prevalência de impacto foi alta; (2) a dimensão mais afetada foi o do desempenho físico comer e apreciar a comi$d a$; (3) a variável sexo foi a que apresentou maior associação com o impacto nos desempenhos diários; (4) o CPOD, quando analisado nas categorias alto, médio e baixo, mostrou associação com a maioria dos desempenhos diários, sendo útil como um indicador de impacto odontológico no desempenho diário.

São necessários estudos com outras faixas etárias e culturas envolvendo indicadores subjetivos, a fim de integrar estimativas de tratamento, ampliar os conceitos de saúde bucal e monitorar a redução do impacto odontológico por meio da atenção em saúde bucal.

\section{Resumo}

O objetivo deste estudo foi investigar a prevalência do impacto bucal no desempenho diário em adultos brasileiros. Uma amostra representativa, composta por 276 funcionários do Departamento Municipal de Limpeza Urbana de Porto Alegre, Rio Grande do Sul, Brasil, entre 35 e 44 anos, responderam a entrevista e permitiram a realização do exame clínico. O Oral Impacts on Daily Performances (OIDP) foi utilizado para avaliar o impacto bucal no desempenho diário. Do total de participantes, 73,6\% tiveram pelo menos um desempenho diário afetado por problemas odontológicos nos últimos seis meses. O mais afetado foi comer e apreciar a comida (48,6\%). O desconforto $(40,6 \%) e$ a insatisfação com a aparência $(31,5 \%)$ foram os sintomas mais prevalentes. A falta de dentes $(21,7 \%)$ e a dor de dente $(20,7 \%)$ foram as principais causas percebidas de impacto no desempenho diário. O OIDP mostrou-se útil para avaliar os impactos odontológicos nas dimensões físicas, psicológicas e sociais do desempenho diário.

Saúde Bucal; Qualidade de Vida; Indicadores; Levantamentos Epidemiológicos

\section{Colaboradores}

A. S. Gomes realizou a pesquisa bibliográfica, a coleta de dados, a análise dos dados e redigiu o artigo. C. Abegg contribuiu na análise dos dados e realizou a revisão do artigo. 


\section{Referências}

1. World Health Organization/Food and Agriculture Organization of the United Nations. Diet, nutrition and the prevention of chronic diseases. Geneva: World Health Organization; 2003. (WHO Technical Report Series, 916).

2. Dolan TA. Identification of appropriate outcomes for an aging population. Spec Care Dentist 1993; 13:35-9.

3. Yewe-Dyer M. The definition of oral health. Br Dent J 1993; 174:224-5.

4. Dubos R. Mirage of health. New York: Harper Colophan; 1979.

5. Laurel AC. A saúde-doença como processo social. In: Nunes E, organizador. Medicina social: aspectos históricos e teóricos. São Paulo: Global Editora; 1983. p. 135-58.

6. Organização Mundial da Saúde. Levantamentos básicos em saúde bucal. 4a Ed. São Paulo: Editora Santos; 1999.

7. Locker D. Measuring oral health: socio-dental indicators. In: Locker D, editor. An introduction to behavioral science \& dentistry. New York/London: Routledge; 1989. p. 73-101.

8. Adulyanon S, Sheiham A. Oral impacts on daily performances. In: Slade GD, editor. Measuring oral health and quality of life. Chapel Hill: School of Dentistry, University of North Carolina; 1997. p. 151-60.

9. Cushing AM, Sheiham A, Maizels J. Developing socio-dental indicators-the social impact of dental disease. Community Dent Health 1986; 3:3-17.

10. Sheiham A. A determinação de necessidades de tratamento odontológico: uma abordagem social. In: Pinto VG, organizador. Saúde bucal coletiva. São Paulo: Editora Santos; 2000. p. 223-50.

11. Srisilapanan P, Sheiham A. Assessing the difference between sociodental and normative approaches to assessing prosthetic dental treatment needs in dentate older people. Gerodontology 2001; 18:2534.

12. Adulyanon S, Vourapukjaru J, Sheiham A. Oral impacts affecting daily performance in a low dental disease Thai population. Community Dent Oral Epidemiol 1996; 24:385-9.

13. World Health Organization. International classification of impairments, disabilities and handcaps. Geneva: World Health Organization; 1980.

14. Masalu JR, Astrom AN. Applicability of an abbreviated version of the oral impacts on daily performances (OIDP) scale for use among Tanzanian students. Community Dent Oral Epidemiol 2003; 31:7-14.
15. Goes PSA. The prevalenceand impact of dental pain in Brazilian schoolchildren and their families [Doctoral Dissertation]. London: Department of Epidemiology and Public Health, University College London; 2001.

16. Cortes MI, Marcenes W, Sheiham A. Impact of traumatic injuries to the permanent teeth on the oral health-related quality of life in 12-14-year-old children. Community Dent Oral Epidemiol 2002; 30:193-8.

17. Sheiham A, Steele JG, Marcenes W, Tsakos G, Finch S, Walls AW. Prevalence of impacts of dental and oral disorders and their effects on eating among older people; a national survey in Great Britain. Community Dent Oral Epidemiol 2001; 29:195203.

18. Robinson PG, Gibson B, Khan FA, Birnbaum W. Validity of two oral health-related quality of life measures. Community Dent Oral Epidemiol 2003; 31:90-9.

19. Srisilapanan P, Sheiham A. The prevalence of dental impacts on daily performances in older people in Northern Thailand. Gerodontology 2001; 18:102-8.

20. Ministério da Saúde. Projeto SB Brasil 2003 - condições de saúde bucal da população brasileira 2002-2003. Resultados principais. Brasília: Ministério da Saúde; 2004.

21. Chavers LS, Gilbert GH, Shelton BJ. Two-year incidence of oral disadvantage, a measure of oral health-related quality of life. Community Dent Oral Epidemiol 2003; 31:21-9.

22. McGrath C, Bedi R. Gender variations in the social impact of oral health. J Ir Dent Assoc 2000; 46:8791.

23. Leao A, Sheiham A. Relation between clinical dental status and subjective impacts on daily living. J Dent Res 1995; 74:1408-13.

24. Slade GD, Spencer AJ, Locker D, Hunt RJ, Strauss RP, Beck JD. Variations in the social impact of oral conditions among older adults in South Australia, Ontario, and North Carolina. J Dent Res 1996; 75:1439-50.

25. Slade GD. The oral health impact profile. In: Slade GD, editor. Measuring oral health and quality of life. Chapel Hill: School of Dentistry, University of North Carolina; 1997. p. 93-104.

26. Nuttall NM, Steele JG, Pine CM, White D, Pitts NB. The impact of oral health on people in the UK in 1998. Br Dent J 2001; 190:121-6.

27. Leao A, Sheiham A. The development of a sociodental measure of dental impacts on daily living. Community Dent Health 1996; 13:22-6.

Recebido em 31/Jan/2006

Versão final reapresentada em 01/Fev/2007 Aprovado em 16/Fev/2007 